Folia Hort. 25/1 (2013): 49-60

DOI: 10.2478/fhort-2013-0006

\title{
Effect of mixed cropping and plant extracts on the growth, yield and pest control of jute (Corchorus olitorius L.)
}

\author{
Adeyela I. Okunlola*, Thomas I. Ofuya
}

Department of Crop Soil and Pest Management

Federal University of Technology

P.M.B. 704, Akure Ondo State, Nigeria

\begin{abstract}
This study investigated the effect of plant extracts (Azadirachta indica and Piper guineense) on the growth and yield of jute (Corchorus olitorius L.) (Cor) under sole and mixed cropping with Amaranthus hybridus (Ama) and Celosia argentea $(\mathrm{Cel})$. The highest percentage increase in plant height, number of leaves, number of branches and stem girth (130.6, 865.0, 220.4 and 114.0\%, respectively) was found in untreated Cor + Cel, cypermethrin treated Cor + Ama, cypermethrin treated Cor and A. indica extract treated Cor + Ama, respectively. The highest percentage increase in shoot weight $(71.0 \%)$, marketable yield $(53.9 \%)$ and total biomass $(51.5 \%)$ was in $A$. indica treated $C$. olitorius. Extract treatment had no effect on the number of branches at four, five and seven weeks after planting (WAP) in Cor + Ama + Cel; four, five and six WAP in Cor + Cel; four and six WAP in Cor + Ama; as well as four and five WAP in Cor. The highest percentage increase in leaf fresh weight was obtained in cypermethrin treated Cor (53.3\%) and Cor + Ama + Cel (52.2\%), while the smallest increase was in $P$. guineense treated Cor (1.1\%) and Cor + Ama (2.5\%). The activity of $A$. indica was independent of time of application while $P$. guineense was not. Among the extract treated groups, the highest percentage increase in shoot weight (71.0\%), marketable yield (53.9\%) and total biomass $(51.5 \%)$ was in $A$. indica treated Cor. The activity of the extracts against insect pests of sole cropped Cor increased significantly $(\mathrm{p}<0.05)$ with increasing extract concentration. The highest efficiency with the use of $P$. guineense was $250 \mathrm{~g} \mathrm{dm}^{-3}$ at four, five and six WAP (94.0, 92.7 and 90.3\%, respectively) and $200 \mathrm{mg} \mathrm{dm}^{-3}$ at seven WAP (81.7\%). In the case of $A$. indica extract, the highest efficiency was found with the use of $200 \mathrm{~g} \mathrm{dm}^{-3}$ at four, six and seven WAP (92.7, 85.3 and $100 \%$, respectively). A. indica extract $\left(250 \mathrm{~g} \mathrm{dm}^{-3}\right)$ treatment gave the highest efficiency at five WAP $(100 \%)$.
\end{abstract}

Key words: insect pests, leafy vegetable, pest management, synthetic insecticides, sustainable agriculture

\section{INTRODUCTION}

The diets of many people in developing countries are dominated by starchy staple foods and are low in animal protein, leading to a high prevalence of malnutrition and stunted growth (Ijarotimi et al. 2003). The situation is worsened by the increase in population, level of poverty and decreasing soil productivity with long-term cropping. These observations underscore the need for appropriate integrated and sustainable management strategies in addressing the situation. Recent developmental efforts have resulted in the spread of information on the value of fresh vegetables in the human diet

\footnotetext{
*Corresponding author.

Tel.: +234 803443 5717; fax: +234803443 5717;

e-mail: aiokunlola@yahoo.co.uk (A.I. Okunlola).
} 
(Oniango 2001). Soft edible structures developed from plant parts other than the seed are usually regarded as vegetables. They are mostly eaten as accompaniments to starchy staples in a raw or cooked form, alone, with meat or fish, in stews, soups or various preparations in which groundnut, melon seed, and palm oil are also commonly added (Ogbalu et al. 2005).

Vegetables like Amaranthus hybridus L., Celosia argentea L., and Corchorus olitorius L. are commonly grown for their edible leaves (leaf vegetables) (Ojeifo et al. 2006). Green leafy vegetables are widely used for food in many countries of the world; they are rich sources of beta carotene, ascorbic acid, minerals and dietary fibre (Negi and Roy 2001, Oboh and Akindahunsi 2004). In Nigeria, crops and cropping systems are diverse owing to large agro-ecological and cultural diversity, which in turn leads to variable cropping patterns. In the traditional Nigerian agricultural system, vegetables are grown in mixtures with cereals, grain legumes and tubers (Ikeorgu 1989, Olasantan 1999, 2005). Although local farmers are favourably disposed to advancements in agricultural practices, the high adaptive index of some of these emerging technologies, coupled with low yield, pesticide toxicity and the changing nature of social-environmental interactions raise a series of unresolved questions on improved productivity (Porter et al. 2003). There has been considerable interest in sustainable production systems for agronomic crops where plant height, stem strength and the number of leaves were major considerations (Eltun et al. 2002, Dapaah et al. 2003, Ngouajio et al. 2003, Jagadamma et al. 2008). Research by various authors show that intercropping increases the size and stability of yields compared to sole cropping (Thangataa and Alavalapatib 2003, HauggaardNielsen et al. 2006, Jahansooz et al. 2007).

Insect pests are also a major factor affecting the productivity of vegetables. The Food and Agriculture Organisation (FAO 1997) reported that the estimated annual losses due to insect pests alone stands at about $15 \%-20 \%$ during production and 18\%-20\% during storage. The Federal Department of Agriculture (Nigeria) estimated that about $60 \%$ of the total food and fibre produced are lost to insect pests (Mohammed 2002). These massive losses underscore the need for a sustainable and environmentally friendly approach for addressing the situation. Synthetic chemical insecticides have proved very effective in the control of insect pests. However, problems associated with chemical insecticides, such as depletion of the ozone layer, health hazards, insect resistance, irregular supplies and cost have led to increased demand for alternatively safer, cheaper and more ecologically friendly methods in the control of these insects. Medicinal plants have received considerable attention as potential sources of alternative materials for use as insecticides and antifeedants. They contain a wide range of bioactive chemicals that are potentially suitable for use in integrated pest management (Pavela 2004, Akendengue et al. 2005, Han et al. 2006, Jovanovic et al. 2007, Georges et al. 2008, Montes-Molina et al. 2008, Javed et al. 2008).

The use of natural plant products as insecticides is particularly important in a country like Nigeria where synthetic insecticides are expensive and not readily available. In addition, farmers are poorly equipped to handle them. Extracts of Azadirachta indica (neem) and Piper guineense have components capable of influencing the physiology and behaviour of a wide range of insects, mites and nematodes (Dhar et al. 1998, Schaaf et al. 2000, Ngono Ngane et al. 2003). The bark, leaf and seed oil are particularly useful parts of the neem tree (Han et al. 2006). Major biologically active components reported in $A$. indica and $P$. guineense include: azadirachtin, triterpenoids, piperolein acids, guineensine, essential oils, sterols and amides, among others (Martins et al. 1998, Schaaf et al. 2000, Siddiqui et al. 2000, Muse et al. 2003, Ngono Ngane et al. 2003, Gopal et al. 2007). Most reports on the use of plant extracts are in relation to stored cereals and legumes, with scant information on vegetables. There is little information on the management of $C$. olitorius using an integrated approach involving mixed cropping and use of $A$. indica and $P$. guineense extracts despite their excellent pharmacological, insecticidal and antifeedant properties. The objective of this research was to study the effects of mixed cropping (with A. hybridus and C. argentea) and plant extracts on the growth and yield performance of $C$. olitorius.

\section{MATERIAL AND METHODS}

\section{Raw material collection and preparation}

The cypermethrin, $C$. olitorius, $A$. hybridus and $C$. argentea used in this study were obtained from the Ondo State Agricultural Development Programme, Akure, Nigeria. Fresh bark samples of $A$. indica were obtained from disease-free trees in a local farm in Akure, Nigeria. Sundried P. guineense was 
obtained from the Oja-Oba market in Akure. The plant parts were authenticated in the Department of Crop Soil and Pest Management of the Federal University of Technology, Akure, Nigeria before use. The vegetable seeds were subjected to hot water treatment to break seed dormancy before sowing.

\section{Preparation of plant extracts}

Fresh bark samples of $A$. indica and sundried $P$. guineense were separately peeled and ground to crumbs in a mortar. Thereafter 100,150, 200 and $250 \mathrm{~g}$ of each were measured and separately soaked in $1 \mathrm{dm}^{3}$ of water, giving four concentrations: 100 $\mathrm{g} \mathrm{dm}^{-3}, 150 \mathrm{~g} \mathrm{dm}^{-3}, 200 \mathrm{~g} \mathrm{dm}^{-3}$ and $250 \mathrm{~g} \mathrm{dm}^{-3}$, respectively. After 24 hours, the extract solutions were sieved with cheesecloth to obtain clear extract solutions. Cypermethrin (synthetic pesticide) was prepared according to the manufacturer's specifications $(0.2 \% \mathrm{v} / \mathrm{v})$.

\section{Site preparation, preparation of seedbed and cultivation of seeds}

The field experiments were conducted on a previously cultivated site during the early season of 2005 and replicated in 2006 at the Teaching and Research Farm of the Federal University of Technology, Akure (327 m above sea level; $\left.7^{\circ} 16^{\prime} \mathrm{N}, 5^{\circ} 12^{\prime} \mathrm{E}\right)$ located in the rainforest zone of south-western Nigeria. The experimental site has luxuriant vegetation, well-drained fertile soils free from iron concentrates and annual rainfall between $1150-2000 \mathrm{~mm}$. The fallow vegetation on the land was manually cleared and the debris packed to the borders to obtain clean seedbeds for sowing the crops. Seedbeds (three per treatment group represent a plot) measuring $2 \mathrm{~m}$ by $2 \mathrm{~m}$ were constructed with a space of $1 \mathrm{~m}$ between the blocks. The vegetable seeds were sown in rows $30 \mathrm{~cm}$ apart and thinned to 20 stands per row with an average spacing of $10 \mathrm{~cm}$ within rows $\left(60\right.$ stands $\left.\mathrm{m}^{-2}\right)$ two weeks later. Weeds were manually removed in the plots at two and five weeks after planting. A composite sample of the soil in the experimental area was collected for routine laboratory analysis. Ten stands were randomly selected per plot and tagged for data collection.

\section{Mixed cropping and treatment with plant extracts}

The crops were planted as follows: plot 1, sole C. olitorius; plot 2, 1:1 rows of $C$. olitorius and $C$. argentea; plot 3, 1:1 rows of $C$. olitorius and A. hybridus and plot 4, 1:1:1 rows of $C$. olitorius, $C$. argentea and $A$. hybridus. The seeds were sown and thinned as described above. Data were collected weekly from four to seven WAP for growth parameters (plant height, number of leaves, stem girth and number of branches per plant). At seven WAP, tagged vegetables in each plot were harvested, washed and the following yield parameters were measured: leaf, shoot and root weights, total biomass and leaf area. Established seedlings in each plot were sprayed weekly (using a Harry brand hand sprayer) with the extracts obtained from $A$. indica and $P$. guineense at $250 \mathrm{~g}$ $\mathrm{dm}^{-3}$ from four to seven WAP. Growth parameters, namely: plant height, number of leaves, number of branches, stem girth, were determined weekly from four to seven WAP. The cypermethrin treated group served as a positive control. Yield parameters, including leaf fresh weight, leaf area, shoot weight, root weight, marketable yield and total biomass, were determined at seven WAP.

\section{Prevalence of insect pests}

Sampling of the pest population on established crop stands from different plots was done four times early in the morning at weekly intervals. At each sampling, the numbers of insects on the plants on each bed were recorded using the direct counting method. Insects responsible for damage found on the plant were collected in sample bottles containing formalin and taken to the laboratory for identification. The insect density on each plot was counted a day before and a day after spraying. Percentage efficiency was determined using the Henderson - Tilton formula (Püntener 1981):

$$
\text { Efficiency }(\%)=\frac{\mathrm{N} \text { in Co before treatment } \times \mathrm{N} \text { in T after treatment }}{\mathrm{N} \text { in Co after treatment } \times \mathrm{N} \text { in T before treatment }} \times 100
$$

where $\mathrm{N}-$ insect population, $\mathrm{T}-$ treated, $\mathrm{Co}-$ control.

\section{Data analysis}

The design of each experiment was a randomised complete block (RCBD) with three replications. Means of triplicate readings were subjected to analysis of variance (ANOVA) while means were separated using Duncan's Multiple Range Test. Relevant data were subjected to correlation and regression analyses. All analyses were carried out using the Statistical Package for Social Scientists (version 10).

\section{RESULTS}

\section{Physical and chemical properties of soil at site of experiment}

Soil $\mathrm{pH}$ and organic matter remained fairly constant after cropping (Tab. 1). Decreases were recorded in 
Table 1. Physical and chemical properties of soil at site of experiment

\begin{tabular}{|c|c|c|c|c|c|c|c|c|c|c|c|}
\hline \multirow[b]{2}{*}{$\begin{array}{l}\text { Time of } \\
\text { analysis }\end{array}$} & \multicolumn{3}{|c|}{ Particle size (\%) } & \multirow[b]{2}{*}{$\mathrm{pH}$} & \multirow{2}{*}{$\begin{array}{c}\text { Organic } \\
\text { matter \% }\end{array}$} & \multicolumn{6}{|c|}{ Exchangeable cation } \\
\hline & sand & silt & clay & & & $\begin{array}{c}\mathrm{N} \\
(\%)\end{array}$ & $\begin{array}{c}\mathrm{P} \\
\left(\mathrm{mg} \mathrm{kg}^{-1}\right)\end{array}$ & $\mathrm{K}$ & $\begin{array}{l}\mathrm{Ca} \\
(\mathrm{cmo}\end{array}$ & $\begin{array}{l}\mathrm{Mg} \\
\left.\mathrm{g}^{-1}\right)\end{array}$ & $\mathrm{Na}$ \\
\hline Before planting & 54 & 18 & 30 & 6.63 & 2.41 & 0.70 & $26.61 \mathrm{~b}$ & $0.76 \mathrm{~b}$ & 2.40 & 1.00 & 0.87 \\
\hline After planting & 54 & 18 & 30 & 6.33 & 2.20 & 0.64 & $10.83 \mathrm{a}$ & $0.51 \mathrm{a}$ & 2.00 & 1.50 & 0.64 \\
\hline
\end{tabular}

Means followed by different letters in the same column are significantly different at $\mathrm{p}=0.05$

Table 2. Growth of C. olitorius intercropped with A. hybridus (Ama) and C. argentea (Cel) before application of plant extracts at 1-3 WAP

\begin{tabular}{lccccc}
\hline Intercropping design & WAP & Cor & Cor + Ama & Cor + Cel & Cor + Ama + Cel \\
\hline \multirow{3}{*}{ No of leaves } & 1 & $20.1 \mathrm{~b} \pm 1.16$ & $20.3 \mathrm{~b} \pm 1.50$ & $20.1 \mathrm{~b} \pm 3.05$ & $17.2 \mathrm{a} \pm 1.33$ \\
& 2 & $27.3 \mathrm{~b} \pm 0.87$ & $25.3 \mathrm{a} \pm 2.27$ & $27.1 \mathrm{~b} \pm 3.97$ & $24.0 \mathrm{a} \pm 2.21$ \\
& 3 & $48.2 \mathrm{~b} \pm 3.94$ & $45.9 \mathrm{a} \pm 2.46$ & $48.8 \mathrm{~b} \pm 7.14$ & $45.7 \mathrm{a} \pm 2.44$ \\
\hline \multirow{2}{*}{ No of branches } & 2 & $5.0 \pm 1.38$ & $5.4 \pm 0.59$ & $5.4 \pm 1.37$ & $4.8 \pm 0.44$ \\
& 3 & $8.2 \pm 0.48$ & $8.6 \pm 0.61$ & $8.6 \pm 0.32$ & $7.3 \pm 0.38$ \\
\hline \multirow{2}{*}{ Stem girth } & 1 & $2.4 \pm 0.01$ & $2.1 \pm 0.19$ & $2.1 \pm 0.18$ & $2.2 \pm 0.19$ \\
& 2 & $2.5 \pm 0.24$ & $2.3 \pm 0.09$ & $2.4 \pm 0.16$ & $2.3 \pm 0.10$ \\
& 3 & $3.5 \pm 0.26$ & $3.6 \pm 0.33$ & $3.5 \pm 0.43$ & $3.3 \pm 0.14$ \\
\hline
\end{tabular}

Means followed by different letters in the same row are significantly different at $\mathrm{p}=0.05$

the sodium, potassium and phosphorus contents of the soil. This observation suggests that cultivation of $C$. olitorius is accompanied by depletion in phosphorus and potassium contents. Table 2 showed the growth parameters of sole and mixed cropped C. olitorius (Cor) at one to three WAP before application of plant extracts. Tables 3 and 4 show the effect of mixed cropping and plant extract and its interaction effects on the growth of $C$. olitorius at four to seven WAP. The highest percentage increase in plant height, number of leaves, number of branches and stem girth (130.6, 865.0, 220.4 and $114.0 \%$, respectively) were observed in untreated $C$. olitorius $+C$. argentea $(\mathrm{Cel})$, cypermethrin treated C. olitorius $+A$. hybridus (Ama), sole cropped cypermethrin treated $C$. olitorius and $A$. indica extract treated $C$. olitorius + Ama, respectively (Figs 1-4).

\section{Growth parameters}

Gradual increases in plant height were recorded in all of the treatment groups. Within the mixed cropped group treated with plant extract, $A$. indica extract treated groups had significantly higher values. The interaction effect was significant in the height of sole and mixed cropped $C$. olitorius. The plant height of $C$. olitorius when intercropped with Ama (Cor + Ama), Cel (Cor + Cel) and Ama $+\mathrm{Cel}(\mathrm{Cor}+\mathrm{Ama}+\mathrm{Cel})$ was significantly higher $(\mathrm{p}<0.05)$ in $A$. indica extract treated groups at four to six WAP. The values obtained for both $P$. guineense and $A$. indica treated mixed cropped $C$. olitorius were significantly higher than the untreated group. However, plant heights were generally higher in sole cropped $C$. olitorius (Tab. 3). Among the untreated groups, the number of leaves was significantly higher when intercropped with Ama (Cor + Ama); the value obtained, 109.1, represent a $75.1 \%$ increase from four to seven WAP. When intercropped with $\mathrm{Cel}(\mathrm{Cor}+\mathrm{Cel})$, the number of leaves was $84 \%$ at seven WAP (representing a 73.4 percent increase). No significant difference ( $p>0.05$ ) was observed in the number of leaves of $A$. indica and $P$. guineense treated sole C. olitorius, $\mathrm{Cor}+\mathrm{Cel}$, Cor $+\mathrm{Ama}+\mathrm{Cel}$ at four WAP. This trend changed at five to seven WAP for sole $C$. olitorius, while the Cor $+\mathrm{Cel}$ with $A$. indica extract treated group had significantly higher values. The highest increase in the number of leaves was in $A$. indica extract treated sole cropped $C$. olitorius (77.8\%; Fig. 2). A similar range was observed when intercropped with celosia and treated with A. indica and P. guineense extract (71.8 and $73.9 \%$, respectively). Cypermethrin treated sole cropped C. olitorius and Cor + Ama had significantly higher percentage increase values $(79.2$ and $86.6 \%$, respectively, (Fig. 4). However, they had the least per cent increase when intercropped with Cel (68.2\%) and Ama + Cel (48.7\%). There were significant increases with intercropping in the number of 


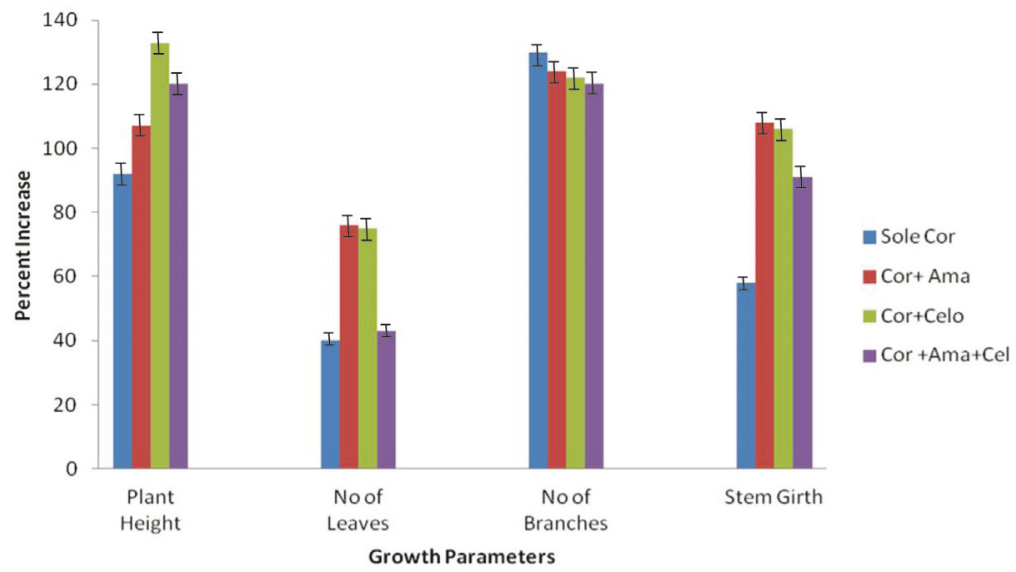

Figure 1. Effect of no spray on the growth of sole and mixed cropped C. olitorius

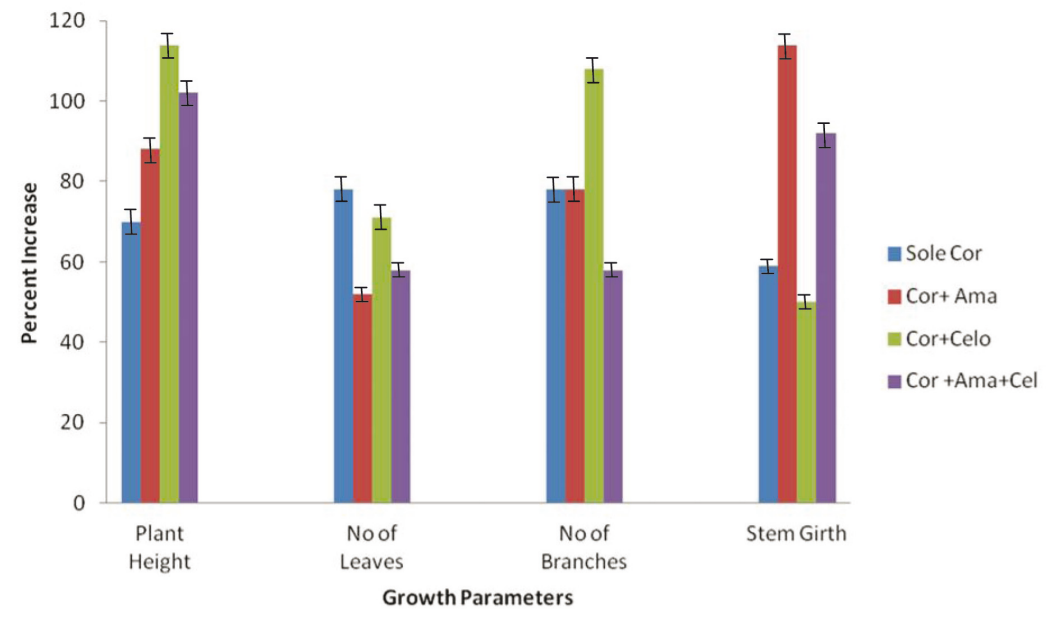

Figure 2. Effect of plant extract (A. indica) $250 \mathrm{~g} \mathrm{dm}^{-3}$ on the growth of C. olitorius

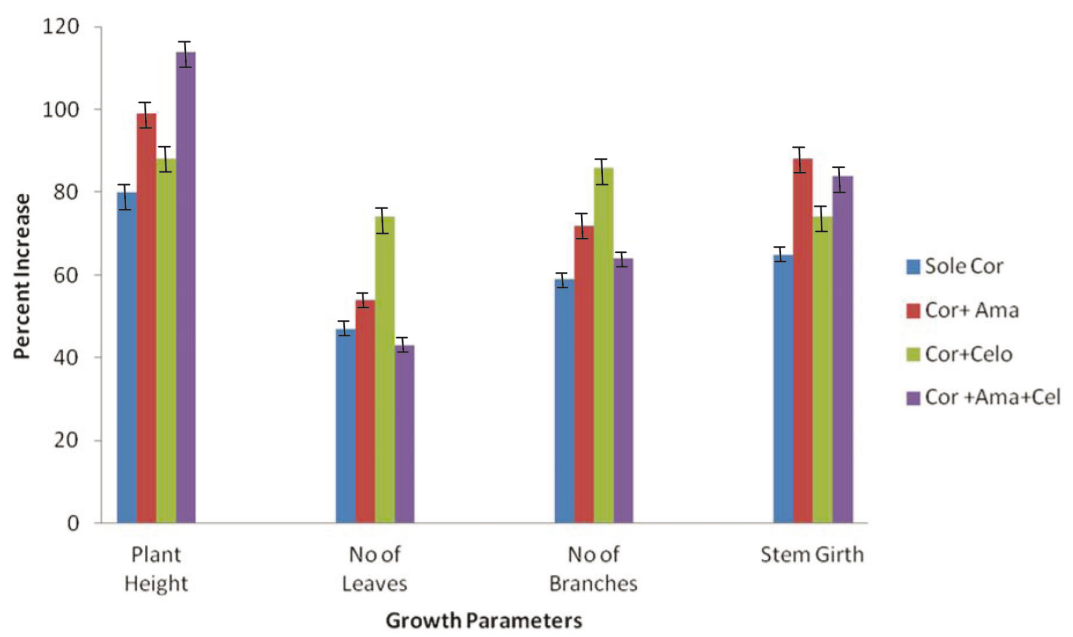

Figure 3. Effect of $P$. guineense $\left(250 \mathrm{~g} \mathrm{dm}^{-3}\right)$ on the growth of sole and mixed cropped $C$. olitorius

branches of $C$. olitorius over the entire planting period. Sole cropped $C$. olitorius had the smallest values throughout the period, while Cor + Ama had the highest values at four, five, and seven WAP.

Extract treatment had no effect on the number of branches at four, five, and seven WAP after planting in C. olitorius mixed cropped with Ama $+\mathrm{Cel}$ (Cor + Ama + Cel); four, five, and six WAP in C. olitorius mixed cropped with Cel (Cor $+\mathrm{Cel})$; four and six WAP in C. olitorius intercropped with Ama (Cor + Ama); and four and five WAP in sole cropped C. olitorius. The highest increase was observed 


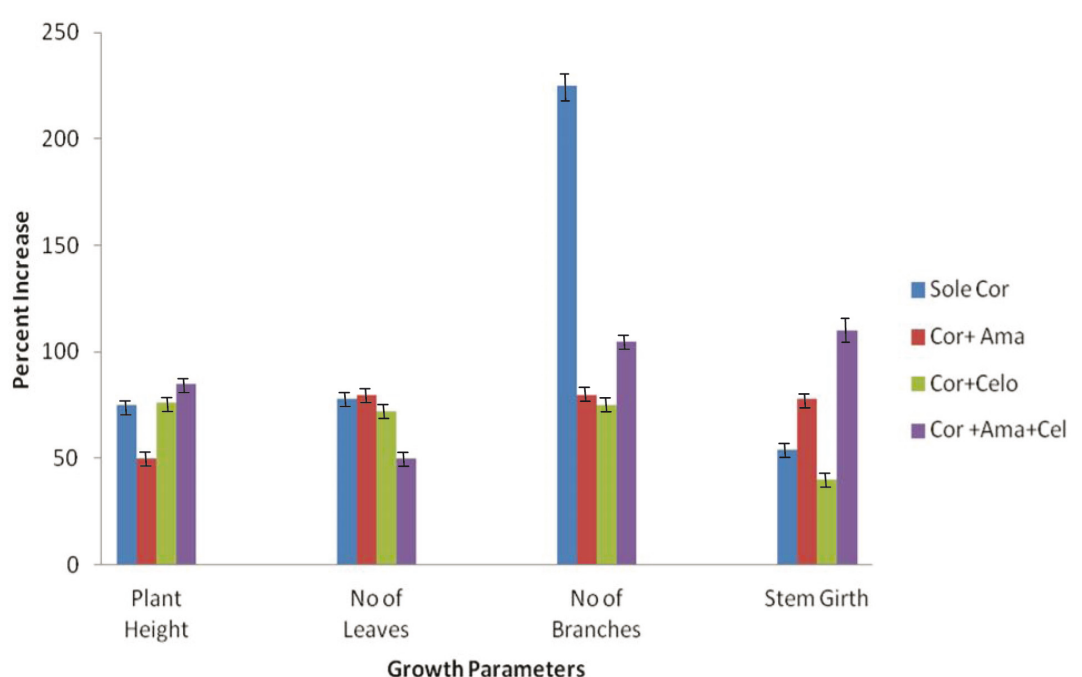

Figure 4. Effect of cypermethrin $(0.2 \% \mathrm{v} / \mathrm{v})$ on the growth of sole and mixed cropped C. olitorius

Table 3. Effect of mixed cropping and plant extract on plant height and number of leaves of C. olitorius at 4-7 WAP

\begin{tabular}{|c|c|c|c|c|c|c|}
\hline Parameter & Intercropping model & Biocide & Week 4 & Week 5 & Week 6 & Week 7 \\
\hline \multirow{16}{*}{$\begin{array}{l}\text { Plant height } \\
(\mathrm{cm})\end{array}$} & \multirow{4}{*}{ C. olitorius } & Untreated & $63.4 c$ & $90.9 c$ & $97.1 \mathrm{~b}$ & $120.4 \mathrm{~d}$ \\
\hline & & A. indica & $80.4 \mathrm{f}$ & $100.7 \mathrm{~d}$ & $120.5 \mathrm{f}$ & $136.6 \mathrm{f}$ \\
\hline & & P. guineense & $75.1 \mathrm{e}$ & $96.3 \mathrm{~cd}$ & $117.3 \mathrm{f}$ & $135.0 \mathrm{f}$ \\
\hline & & Cypermethrin & $83.1 \mathrm{f}$ & $111.3 \mathrm{e}$ & $121.4 \mathrm{f}$ & $143.5 \mathrm{~g}$ \\
\hline & \multirow{4}{*}{$\begin{array}{l}\text { C. olitorius + } \\
\text { A. hybridus }\end{array}$} & Untreated & $53.5 \mathrm{~b}$ & $74.8 \mathrm{ab}$ & $90.4 \mathrm{~b}$ & $109.0 \mathrm{c}$ \\
\hline & & A. indica & $65.0 \mathrm{~cd}$ & $93.4 \mathrm{c}$ & $109.4 \mathrm{~cd}$ & $122.5 \mathrm{de}$ \\
\hline & & P. guineense & $60.5 \mathrm{bc}$ & $81.7 \mathrm{~b}$ & $100.2 \mathrm{bc}$ & $119.8 \mathrm{~d}$ \\
\hline & & Cypermethrin & $81.9 \mathrm{f}$ & $100.7 \mathrm{~d}$ & $112.0 \mathrm{de}$ & $122.5 \mathrm{de}$ \\
\hline & \multirow{4}{*}{$\begin{array}{l}\text { C. olitorius + } \\
\text { C. argentea }\end{array}$} & Untreated & $46.4 \mathrm{a}$ & $80.0 \mathrm{~b}$ & $90.1 \mathrm{~b}$ & $106.9 \mathrm{~b}$ \\
\hline & & A. indica & $59.9 \mathrm{bc}$ & $102.4 \mathrm{~d}$ & $109.7 \mathrm{de}$ & $128.2 \mathrm{e}$ \\
\hline & & P. guineense & $59.1 \mathrm{bc}$ & $100.1 \mathrm{~d}$ & $102.6 \mathrm{~cd}$ & $109.8 \mathrm{c}$ \\
\hline & & Cypermethrin & $75.3 \mathrm{e}$ & $103.2 \mathrm{~d}$ & $116.3 \mathrm{e}$ & $129.5 \mathrm{e}$ \\
\hline & \multirow{4}{*}{$\begin{array}{l}\text { C. olitorius }+ \\
\text { A. hybridus }+ \\
\text { C. argentea }\end{array}$} & Untreated & $42.73 \mathrm{a}$ & $69.3 \mathrm{a}$ & $76.7 \mathrm{a}$ & $92.7 \mathrm{a}$ \\
\hline & & A. indica & $66.9 \mathrm{~cd}$ & $100.0 \mathrm{~d}$ & $113.6 \mathrm{e}$ & $135.0 \mathrm{f}$ \\
\hline & & P. guineense & $59.0 \mathrm{bc}$ & $91.4 \mathrm{c}$ & $104.0 \mathrm{~cd}$ & $126.5 \mathrm{e}$ \\
\hline & & Cypermethrin & $70.9 \mathrm{e}$ & $100.5 \mathrm{~d}$ & $122.6 \mathrm{f}$ & $135.7 \mathrm{f}$ \\
\hline \multirow{16}{*}{ No of leaves } & \multirow{4}{*}{ C. olitorius } & Untreated & $58.6 \mathrm{~b}$ & $69.7 \mathrm{a}$ & $94.7 \mathrm{a}$ & $80.9 \mathrm{a}$ \\
\hline & & A. indica & $70.8 \mathrm{e}$ & $100.3 \mathrm{~d}$ & $99.1 \mathrm{ab}$ & 125.9 e \\
\hline & & P. guineense & $69.5 \mathrm{de}$ & $79.4 \mathrm{~b}$ & $95.1 \mathrm{a}$ & $101.6 \mathrm{~b}$ \\
\hline & & Cypermethrin & $73.3 \mathrm{e}$ & $106.2 \mathrm{e}$ & $112.1 \mathrm{de}$ & $131.3 \mathrm{f}$ \\
\hline & \multirow{4}{*}{$\begin{array}{l}\text { C. olitorius + } \\
\text { A. hybridus }\end{array}$} & Untreated & $62.3 c$ & $79.4 \mathrm{~b}$ & $99.3 \mathrm{ab}$ & $109.1 \mathrm{c}$ \\
\hline & & A. indica & $80.8 \mathrm{f}$ & $93.5 \mathrm{c}$ & $109.6 \mathrm{~cd}$ & $123.7 \mathrm{e}$ \\
\hline & & P. guineense & $74.8 \mathrm{e}$ & $86.4 \mathrm{c}$ & $101.4 \mathrm{bc}$ & $116.3 \mathrm{c}$ \\
\hline & & Cypermethrin & $83.5 \mathrm{f}$ & $95.1 \mathrm{~cd}$ & $109.5 \mathrm{~cd}$ & $155.7 \mathrm{~g}$ \\
\hline & \multirow{4}{*}{$\begin{array}{l}\text { C. olitorius + } \\
\text { C. argentea }\end{array}$} & Untreated & $48.5 \mathrm{a}$ & $86.7 \mathrm{c}$ & $104.8 \mathrm{~cd}$ & $84.0 \mathrm{a}$ \\
\hline & & A. indica & $65.0 \mathrm{~cd}$ & $116.5 \mathrm{f}$ & $116.6 \mathrm{e}$ & $111.7 \mathrm{c}$ \\
\hline & & P. guineense & $62.3 c$ & $93.2 \mathrm{c}$ & $106.3 \mathrm{~cd}$ & $108.3 \mathrm{c}$ \\
\hline & & Cypermethrin & $79.8 \mathrm{f}$ & $165.6 \mathrm{~h}$ & $167.7 \mathrm{~h}$ & $133.7 \mathrm{f}$ \\
\hline & \multirow{4}{*}{$\begin{array}{l}\text { C. olitorius + } \\
\text { A. hybridus }+ \\
\text { C. argentea }\end{array}$} & Untreated & $55.9 \mathrm{~b}$ & $91.9 \mathrm{c}$ & $94.1 \mathrm{a}$ & $80.1 \mathrm{a}$ \\
\hline & & A. indica & $76.5 \mathrm{ef}$ & $97.9 \mathrm{~d}$ & $123.1 \mathrm{f}$ & $120.1 \mathrm{de}$ \\
\hline & & P. guineense & $72.0 \mathrm{e}$ & $99.1 \mathrm{~d}$ & $116.3 \mathrm{e}$ & $103.0 \mathrm{bc}$ \\
\hline & & Cypermethrin & $81.1 \mathrm{f}$ & $138.2 \mathrm{~g}$ & $145.1 \mathrm{~g}$ & $120.6 \mathrm{de}$ \\
\hline
\end{tabular}

Means followed by different letters in each column for a given trait are significantly different at $\mathrm{p}=0.05$ 
Table 4. Effect of mixed cropping and plant extract on number of branches and stem girth of C. olitorius at 4-7 WAP

\begin{tabular}{|c|c|c|c|c|c|c|}
\hline Parameter & Intercropping model & Biocide & Week 4 & Week 5 & Week 6 & Week 7 \\
\hline \multirow{16}{*}{ No of branches } & \multirow{4}{*}{ C. olitorius } & Untreated & $7.3 \mathrm{a}$ & $12.0 \mathrm{~b}$ & $15.0 \mathrm{~b}$ & $16.5 \mathrm{a}$ \\
\hline & & A. indica & $12.9 \mathrm{de}$ & $16.0 \mathrm{e}$ & $21.1 \mathrm{f}$ & $23.0 \mathrm{f}$ \\
\hline & & P. guineense & $12.0 \mathrm{~d}$ & $15.3 \mathrm{c}$ & $16.8 \mathrm{c}$ & $19.1 \mathrm{~d}$ \\
\hline & & Cypermethrin & $13.4 \mathrm{e}$ & $21.6 \mathrm{f}$ & $25.7 \mathrm{~g}$ & $42.9 \mathrm{i}$ \\
\hline & \multirow{4}{*}{$\begin{array}{l}\text { C. olitorius + } \\
\text { A. hybridus }\end{array}$} & Untreated & $9.5 \mathrm{c}$ & $14.0 \mathrm{c}$ & $16.6 \mathrm{c}$ & $20.6 \mathrm{e}$ \\
\hline & & A. indica & $13.4 \mathrm{e}$ & $16.3 \mathrm{e}$ & $18.1 \mathrm{~d}$ & $23.9 \mathrm{f}$ \\
\hline & & P. guineense & $12.1 \mathrm{~d}$ & $14.5 \mathrm{c}$ & $18.1 \mathrm{~d}$ & $20.8 \mathrm{e}$ \\
\hline & & Cypermethrin & $15.3 \mathrm{f}$ & $16.3 \mathrm{e}$ & $18.4 \mathrm{~d}$ & $27.8 \mathrm{~g}$ \\
\hline & \multirow{4}{*}{$\begin{array}{l}\text { C. olitorius }+ \\
\text { C. argentea }\end{array}$} & Untreated & $8.3 \mathrm{~b}$ & $12.8 \mathrm{~b}$ & $16.9 \mathrm{c}$ & $17.7 \mathrm{~b}$ \\
\hline & & A. indica & $10.3 \mathrm{c}$ & $17.1 \mathrm{e}$ & $18.4 \mathrm{~d}$ & $21.4 \mathrm{e}$ \\
\hline & & P. guineense & $9.9 \mathrm{c}$ & $15.4 \mathrm{~d}$ & $18.2 \mathrm{~d}$ & $18.2 \mathrm{c}$ \\
\hline & & Cypermethrin & $13.2 \mathrm{e}$ & $17.3 \mathrm{e}$ & $19.4 \mathrm{e}$ & $22.9 \mathrm{f}$ \\
\hline & \multirow{4}{*}{$\begin{array}{l}\text { C. olitorius }+ \\
\text { A. hybridus }+ \\
\text { C. argentea }\end{array}$} & Untreated & $8.3 \mathrm{~b}$ & $11.5 \mathrm{a}$ & $12.4 \mathrm{a}$ & $17.5 \mathrm{~b}$ \\
\hline & & A. indica & $13.4 \mathrm{e}$ & $16.5 \mathrm{e}$ & $20.7 \mathrm{f}$ & $21.0 \mathrm{e}$ \\
\hline & & P. guineense & $12.7 \mathrm{~d}$ & $15.1 \mathrm{c}$ & $12.4 \mathrm{a}$ & $20.7 \mathrm{e}$ \\
\hline & & Cypermethrin & $14.5 \mathrm{f}$ & $20.5 \mathrm{f}$ & $24.2 \mathrm{~g}$ & $29.5 \mathrm{~h}$ \\
\hline \multirow{16}{*}{$\begin{array}{l}\text { Stem girth } \\
(\mathrm{mm})\end{array}$} & \multirow{4}{*}{ C. olitorius } & Untreated & $4.5 \mathrm{~d}$ & $4.9 \mathrm{a}$ & $5.5 \mathrm{~b}$ & $6.9 \mathrm{~b}$ \\
\hline & & A. indica & $4.8 \mathrm{~d}$ & $6.2 \mathrm{ef}$ & $7.0 \mathrm{de}$ & $7.7 \mathrm{~d}$ \\
\hline & & P. guineense & $4.5 \mathrm{~d}$ & $5.3 \mathrm{c}$ & $6.7 \mathrm{~d}$ & $7.5 \mathrm{c}$ \\
\hline & & Cypermethrin & $5.4 \mathrm{e}$ & $6.4 \mathrm{f}$ & $7.1 \mathrm{e}$ & $8.2 \mathrm{e}$ \\
\hline & \multirow{4}{*}{$\begin{array}{l}\text { C. olitorius + } \\
\text { A. hybridus }\end{array}$} & Untreated & $3.9 \mathrm{ab}$ & $4.7 \mathrm{a}$ & $5.9 \mathrm{bc}$ & $7.4 \mathrm{c}$ \\
\hline & & A. indica & $4.3 \mathrm{~cd}$ & $5.3 \mathrm{c}$ & $7.1 \mathrm{e}$ & $9.2 \mathrm{ef}$ \\
\hline & & P. guineense & $4.2 \mathrm{bc}$ & $4.9 \mathrm{ab}$ & $6.1 \mathrm{c}$ & $7.8 \mathrm{~d}$ \\
\hline & & Cypermethrin & $5.7 \mathrm{e}$ & $5.9 \mathrm{de}$ & $7.1 \mathrm{de}$ & $10.1 \mathrm{f}$ \\
\hline & \multirow{4}{*}{$\begin{array}{l}\text { C. olitorius }+ \\
\text { C. argentea }\end{array}$} & Untreated & $3.8 \mathrm{ab}$ & $5.1 \mathrm{bc}$ & $6.6 \mathrm{~d}$ & $7.2 \mathrm{c}$ \\
\hline & & A. indica & $4.7 \mathrm{~d}$ & $5.4 \mathrm{c}$ & $6.8 \mathrm{~d}$ & $7.2 \mathrm{c}$ \\
\hline & & P. guineense & $4.1 \mathrm{bc}$ & $5.2 \mathrm{c}$ & $6.7 \mathrm{~d}$ & $7.1 \mathrm{bc}$ \\
\hline & & Cypermethrin & $5.3 \mathrm{e}$ & $6.9 \mathrm{~g}$ & $6.9 \mathrm{de}$ & $7.3 \mathrm{c}$ \\
\hline & \multirow{4}{*}{$\begin{array}{l}\text { C. olitorius + } \\
\text { A. hybridus }+ \\
\text { C. argentea }\end{array}$} & Untreated & $3.2 \mathrm{a}$ & $4.5 \mathrm{a}$ & $4.9 \mathrm{a}$ & $5.6 \mathrm{a}$ \\
\hline & & A. indica & $4.6 \mathrm{~d}$ & $6.0 \mathrm{e}$ & $7.6 \mathrm{f}$ & $8.9 \mathrm{e}$ \\
\hline & & P. guineense & $4.2 \mathrm{~cd}$ & $5.5 \mathrm{~cd}$ & $7.1 \mathrm{de}$ & $7.8 \mathrm{~d}$ \\
\hline & & Cypermethrin & $4.9 \mathrm{~d}$ & $6.8 \mathrm{~g}$ & $8.1 \mathrm{~g}$ & $10.2 \mathrm{f}$ \\
\hline
\end{tabular}

Means followed by different letters in each column for a given trait are significantly different at $\mathrm{p}=0.05$

in untreated sole cropped C. olitorius (125.5\%), while the other untreated mixed cropped groups had percentage increases between 111.3-116.2\%. Among the extract treated groups (Figs 2 and 3), A. indica treated sole C. olitorius, Cor + Ama and Cor + Cel had the highest percentage increase (78.3, 78.1 and $107.2 \%$, respectively).

The lowest percentage increase was observed in sole cropped $P$. guineense treated $C$. olitorius (59.5\%, Fig. 3) and A. indica treated Cor + Ama + Cel (56.7\%, Fig. 2). The number of branches was highest in cypermethrin treated Cor + Ama (27.80; 81.3\% increase), Cor + Ama + Cel (29; 104.1\% increase) and sole cropped Cor (42.9; 220.4\%). Stem girth was lower in untreated mixed cropped C. olitorius. However, this trend was reversed in the plant extract treated groups. The highest percentage increases were observed in $P$. guineense (sole Cor, 65:6; Cor + Cel, 74.4\%), and A. indica treated Cor + Ama + Cel. The cypermethrin treated group had significantly higher stem girth than the others (Tab. 4). Table 5 shows the yield of sole and mixed cropped $C$. olitorius treated with plant extracts. Leaf fresh weight (23.9 g), leaf area $\left(168.9 \mathrm{~mm}^{2}\right)$, shoot weight $(54.6 \mathrm{~g})$, marketable yield $\left(87.4 \mathrm{~g} \mathrm{~m}^{-2}\right)$ and total biomass $(64.4 \mathrm{~g})$ were significantly higher in untreated Cor + Ama. The lowest shoot weight (39.4 g), root weight (5.97 g), marketable yield $\left(63.8 \mathrm{~g} \mathrm{~m}^{-2}\right)$ and total biomass $\left(56.8 \mathrm{~g} \mathrm{~m}^{-2}\right)$ were obtained in untreated Cor + Ama + Cel. Cypermethrin treated sole and mixed cropped $C$. olitorius had the highest values of all of 
Table 5. Effect of mixed cropping and plant extract on yield of C. olitorius at 7 WAP

\begin{tabular}{|c|c|c|c|c|c|c|c|}
\hline Intercropping design & Biocide & $\begin{array}{c}\text { Leaf fresh } \\
\text { weight }\end{array}$ & $\begin{array}{l}\text { Leaf } \\
\text { area }\end{array}$ & $\begin{array}{c}\text { Shoot } \\
\text { weight }\end{array}$ & $\begin{array}{c}\text { Root } \\
\text { weight }\end{array}$ & $\begin{array}{c}\text { Marketable } \\
\text { yield }\end{array}$ & $\begin{array}{c}\text { Total } \\
\text { biomass }\end{array}$ \\
\hline \multirow{4}{*}{ C. olitorius } & Untreated & $18.9 \mathrm{ab}$ & $128.1 \mathrm{c}$ & $49.5 \mathrm{~b}$ & $8.7 \mathrm{c}$ & $77.8 \mathrm{~b}$ & $58.9 \mathrm{ab}$ \\
\hline & A. indica & $23.5 \mathrm{~d}$ & $170.9 \mathrm{f}$ & $84.7 \mathrm{f}$ & $10.7 \mathrm{e}$ & $119.8 \mathrm{~g}$ & $89.2 \mathrm{~g}$ \\
\hline & P. guineense & $19.1 \mathrm{~b}$ & $162.1 \mathrm{e}$ & $59.4 \mathrm{~d}$ & $9.4 \mathrm{~d}$ & $87.2 \mathrm{c}$ & $70.4 \mathrm{~d}$ \\
\hline & Cypermethrin & $29.0 \mathrm{~g}$ & $216.7 \mathrm{~h}$ & $85.3 \mathrm{f}$ & $11.6 \mathrm{f}$ & $125.0 \mathrm{~h}$ & $96.2 \mathrm{~h}$ \\
\hline \multirow{4}{*}{$\begin{array}{l}\text { C. olitorius + } \\
\text { A. hybridus }\end{array}$} & Untreated & $23.9 \mathrm{~d}$ & $168.9 \mathrm{e}$ & $54.7 \mathrm{c}$ & $8.4 \mathrm{c}$ & $87.5 \mathrm{c}$ & $64.4 \mathrm{bc}$ \\
\hline & A. indica & $30.5 \mathrm{~g}$ & $182.1 \mathrm{~g}$ & $60.8 \mathrm{~d}$ & $9.4 \mathrm{~d}$ & 99.4 de & $69.0 \mathrm{~cd}$ \\
\hline & P. guineense & $24.5 \mathrm{~d}$ & $167.0 \mathrm{e}$ & $59.5 \mathrm{~d}$ & $9.0 \mathrm{~cd}$ & $93.6 \mathrm{~d}$ & $67.9 \mathrm{~cd}$ \\
\hline & Cypermethrin & $30.0 \mathrm{~g}$ & $212.6 \mathrm{lh}$ & $64.0 \mathrm{e}$ & $11.6 \mathrm{f}$ & $105.6 \mathrm{f}$ & $77.1 \mathrm{e}$ \\
\hline \multirow{4}{*}{$\begin{array}{l}\text { C. olitorius }+ \\
\text { C. argentea }\end{array}$} & Untreated & $21.7 \mathrm{c}$ & $99.9 \mathrm{a}$ & $47.7 \mathrm{~b}$ & $6.7 \mathrm{ab}$ & $76.3 \mathrm{~b}$ & $57.6 \mathrm{a}$ \\
\hline & A. indica & $26.5 \mathrm{e}$ & $190.7 \mathrm{~g}$ & $67.1 \mathrm{e}$ & $10.8 \mathrm{ef}$ & 102.8 ef & $72.3 \mathrm{~d}$ \\
\hline & P. guineense & $24.0 \mathrm{~d}$ & $141.8 \mathrm{~d}$ & $52.3 \mathrm{c}$ & $6.0 \mathrm{~b}$ & $87.1 \mathrm{c}$ & $63.1 \mathrm{~b}$ \\
\hline & Cypermethrin & $29.0 \mathrm{fg}$ & $206.9 \mathrm{~h}$ & $70.1 \mathrm{e}$ & 10.9 ef & $107.9 \mathrm{f}$ & $83.9 \mathrm{f}$ \\
\hline \multirow{4}{*}{$\begin{array}{l}\text { C. olitorius }+ \\
\text { A. hybridus }+ \\
\text { C. argentea }\end{array}$} & Untreated & $18.4 \mathrm{a}$ & $113.1 \mathrm{~b}$ & $39.4 \mathrm{a}$ & $6.0 \mathrm{a}$ & $63.8 \mathrm{a}$ & $56.8 \mathrm{a}$ \\
\hline & A. indica & $24.3 \mathrm{~d}$ & $147.7 \mathrm{~d}$ & $64.1 \mathrm{e}$ & $9.7 \mathrm{~d}$ & $99.6 \mathrm{de}$ & $71.7 \mathrm{~d}$ \\
\hline & P. guineense & $26.3 \mathrm{e}$ & $120.5 \mathrm{~b}$ & $63.7 \mathrm{e}$ & $9.6 \mathrm{~d}$ & $98.0 \mathrm{~d}$ & $68.9 \mathrm{~cd}$ \\
\hline & Cypermethrin & $28.0 \mathrm{f}$ & $171.5 \mathrm{f}$ & $82.2 \mathrm{f}$ & $13.9 \mathrm{~g}$ & $124.1 \mathrm{~h}$ & $111.7 \mathrm{i}$ \\
\hline
\end{tabular}

Means followed by different letters in each column are significantly different at $\mathrm{p}=0.05$

Table 6. Efficiency (\%) of plant extract concentration in the control of insect pests infesting C. olitorius

\begin{tabular}{lccccc}
\hline & Biocide & \multicolumn{4}{c}{ Weeks after planting (WAP) } \\
\cline { 2 - 6 }$\left(\mathrm{mg} \mathrm{dm}^{-3}\right)$ & 4 & 5 & 6 & 7 \\
\hline \multirow{3}{*}{ P. guineense } & 250 & $94.0 \mathrm{~b} \pm 6.00$ & $92.7 \mathrm{~d} \pm 7.33$ & $90.3 \mathrm{bc} \pm 4.91$ & $78.0 \mathrm{bc} \pm 1.53$ \\
& 200 & $86.0 \mathrm{~b} \pm 7.02$ & $78.7 \mathrm{bc} \pm 12.13$ & $85.0 \mathrm{ab} \pm 2.08$ & $81.7 \mathrm{bc} \pm 4.41$ \\
& 150 & $51.3 \mathrm{a} \pm 5.24$ & $23.0 \mathrm{a} \pm 14.57$ & $42.3 \mathrm{a} \pm 16.91$ & $58.7 \mathrm{ab} \pm 9.35$ \\
A. indica & 100 & $60.7 \mathrm{a} \pm 7.86$ & $48.0 \mathrm{abc} \pm 13.65$ & $55.7 \mathrm{ab} \pm 3.18$ & $47.7 \mathrm{a} \pm 8.67$ \\
\hline & 250 & $90.7 \mathrm{bc} \pm 4.70$ & $100.0 \mathrm{~d}$ & $63.3 \mathrm{ab} \pm 31.80$ & $94.7 \mathrm{c} \pm 5.33$ \\
& 200 & $92.7 \mathrm{bc} \pm 7.33$ & $83.0 \mathrm{~cd} \pm 8.50$ & $85.3 \mathrm{ab} \pm 7.36$ & $100.0 \mathrm{c}$ \\
& 150 & $70.3 \mathrm{a} \pm 4.91$ & $45.7 \mathrm{abc} \pm 15.72$ & $73.0 \mathrm{ab} \pm 3.46$ & $64.7 \mathrm{ab} \pm 10.09$ \\
& 100 & $72.0 \mathrm{ab} \pm 14.05$ & $37.0 \mathrm{ab} \pm 21.50$ & $67.0 \mathrm{ab} \pm 1.73$ & $59.0 \mathrm{ab} \pm 9.50$ \\
\hline
\end{tabular}

Means followed by different letters in each column are significantly different at $\mathrm{p}=0.05$

the evaluated yields. $A$. indica treated sole cropped C. olitorius, Cor + Ama and Cor + Cel had significantly higher leaf fresh weight than $P$. guineense treated groups. Amongst the $A$. indica treated groups, mixed cropped Cor had higher values for all of the evaluated yields. The highest percentage increase in leaf fresh weight was obtained in cypermethrin treated sole cropped $C$. olitorius (53.3\%) and Cor + Ama + Cel (52.2\%), while the lowest increase was observed in $P$. guineense treated sole cropped Cor $(1.06 \%)$ and Cor + Ama (2.5\%). Among the extract treated groups, the highest percentage increase in shoot weight $(71 \%)$, marketable yield $(53.9 \%)$ and total biomass $(51.5 \%)$ was observed in $A$. indica treated sole cropped $C$. olitorius. Tables 6-8 showed the efficiency of $A$. indica and $P$. guineense extracts and mixed cropping in the control of $C$. olitorius insect pests. During both seasons, cropping combinations influenced the infestation and severity of the three leafy vegetable pests. There was reduced pest infestation in the Cor + Ama + Cel mixed crop compared with Cor $+\mathrm{Cel}$ and Cor + Ama. Infestation was higher in the sole cropped C. olitorius. Podagrica sp., Sylepta sp. Dsysdercus sp., and Zonocerus sp. were the most abundant pests in the cropping systems studied. The activity of the extracts against pests infesting sole cropped Cor increased significantly $(\mathrm{p}<0.05)$ with increasing extract concentration. The highest efficiency with the use of $P$. guineense was $250 \mathrm{mg} \mathrm{dm}^{-3}$ at four, five and six WAP (94, 92.7 and 90.3\%, respectively) and $200 \mathrm{mg} \mathrm{dm}^{-3}$ at seven WAP (81.6\%). With $A$. indica extract, the highest efficiency was 
Table 7. Impact of plant extracts and mixed cropping on insect pest abundance of $C$. olitorius depending on the year and time (weeks) after planting (WAP)

\begin{tabular}{lccccccccc}
\hline & \multirow{2}{*}{ Parameters } & \multicolumn{9}{c}{2005} & \multicolumn{5}{c}{2006} \\
\cline { 3 - 10 } & & 4 & 5 & 6 & 7 & 4 & 5 & 6 & 7 \\
\hline \multirow{3}{*}{ Plant } & Untreated & $4.4 \mathrm{c}$ & $3.9 \mathrm{c}$ & $3.4 \mathrm{c}$ & $3.2 \mathrm{c}$ & $10.3 \mathrm{c}$ & $8.9 \mathrm{~d}$ & $9.5 \mathrm{c}$ & $6.2 \mathrm{c}$ \\
extract & A. azadirachta & $3.8 \mathrm{bc}$ & $2.5 \mathrm{~b}$ & $1.8 \mathrm{~b}$ & $1.4 \mathrm{~b}$ & $5.1 \mathrm{~b}$ & $2.3 \mathrm{~b}$ & $1.0 \mathrm{a}$ & $0.8 \mathrm{a}$ \\
& P. guineense & $3.1 \mathrm{~b}$ & $2.4 \mathrm{~b}$ & $2.1 \mathrm{~b}$ & $1.7 \mathrm{~b}$ & $5.9 \mathrm{~b}$ & $4.7 \mathrm{c}$ & $2.2 \mathrm{~b}$ & $1.4 \mathrm{~b}$ \\
& Cypermethrin & $2.3 \mathrm{a}$ & $1.3 \mathrm{a}$ & $1.1 \mathrm{a}$ & $0.8 \mathrm{a}$ & $3.9 \mathrm{a}$ & $1.3 \mathrm{a}$ & $0.8 \mathrm{a}$ & $0.8 \mathrm{a}$ \\
\hline \multirow{3}{*}{ Crop } & Cor. & $18.0 \mathrm{c}$ & $13.0 \mathrm{c}$ & $8.5 \mathrm{~b}$ & $6.7 \mathrm{c}$ & $25.3 \mathrm{~d}$ & $18.3 \mathrm{c}$ & $14.9 \mathrm{c}$ & $10.9 \mathrm{~d}$ \\
mixtures & Cor + Ama & $10.9 \mathrm{a}$ & $8.7 \mathrm{~b}$ & $7.0 \mathrm{ab}$ & $6.3 \mathrm{c}$ & $17.0 \mathrm{~b}$ & $12.0 \mathrm{~b}$ & $10.7 \mathrm{~b}$ & $7.6 \mathrm{c}$ \\
& Cor + Cel & $12.3 \mathrm{~b}$ & $7.7 \mathrm{a}$ & $6.3 \mathrm{a}$ & $5.7 \mathrm{~b}$ & $19.7 \mathrm{c}$ & $12.3 \mathrm{~b}$ & $9.2 \mathrm{~b}$ & $5.0 \mathrm{~b}$ \\
& Cor + Cel + Ama & $9.9 \mathrm{a}$ & $8.8 \mathrm{~b}$ & $6.6 \mathrm{a}$ & $4.2 \mathrm{a}$ & $10.9 \mathrm{a}$ & $8.3 \mathrm{a}$ & $6.9 \mathrm{a}$ & $3.3 \mathrm{a}$ \\
\hline
\end{tabular}

Means followed by different letters in each column for a given trait are significantly different at $\mathrm{p}=0.05$

Ama - A. hybridus, Cel - C. argentea, Cor - C. olitorius

Table 8. Pearson correlation matrix showing the relationship between extract efficiencies at 4-7 WAP and Piper guineense or Azadirachta indica extract concentration

\begin{tabular}{|c|c|c|c|c|c|c|}
\hline & \multirow{2}{*}{$\begin{array}{c}\text { Extract } \\
\left(\mathrm{mg} \mathrm{dm}^{-3}\right)\end{array}$} & \multirow{2}{*}{ Time } & \multicolumn{4}{|c|}{ Extract concentration $\left(\mathrm{mg} \mathrm{dm}^{-3}\right)$} \\
\hline & & & 250 & 200 & 150 & 100 \\
\hline \multirow{4}{*}{ P. guineense } & 250 & 0.89 & 1.00 & & & \\
\hline & 200 & 0.26 & -0.22 & 1.00 & & \\
\hline & 150 & -0.35 & 0.60 & -0.58 & 1.00 & \\
\hline & 100 & 0.64 & -0.59 & -0.90 & -0.27 & 1.00 \\
\hline \multirow{4}{*}{ A. indica } & 250 & 0.19 & 1.00 & & & \\
\hline & 200 & -0.41 & -0.28 & 1.00 & & \\
\hline & 150 & -0.11 & 0.70 & -0.39 & 1.00 & \\
\hline & 100 & 0.08 & 0.56 & -0.42 & -0.97 & 1.00 \\
\hline
\end{tabular}

observed with the use of $200 \mathrm{mg} \mathrm{dm}^{-3}$ at four, six and seven WAP (92.7, 85 and 100\%, respectively). $250 \mathrm{mg} \mathrm{dm}^{-3} A$. indica extract treatment gave the highest efficiency at five WAP (100\%). Results in Table 8 show that the activity of $P$. guineense at 100 and $250 \mathrm{mg} \mathrm{dm}^{-3}$ is time-dependent (WAP) with $\mathrm{r}$ (Pearson correlation coefficient) values 0.64 and 0.89 , respectively. Results obtained with $A$. indica extracts suggest that the activity of A. indica extract is independent of time of application (whether four, five, six, or seven WAP) with the use of 100,150 and $250 \mathrm{mg} \mathrm{dm}^{-3}(\mathrm{r}=0.08,0.11$ and 0.19 , respectively). Low $\mathrm{r}$ values indicate little or no relationship between the parameters studied. A strong positive relationship was observed between percent efficiency and application times (WAP) in $P$. guineense treated Cor.

\section{DISCUSSION}

\section{Growth, development and yield of Corchorus olitorius under sole and mixed cropping}

The significantly higher plant height of $C$. olitorius in sole cropping compared to mixed may be due to reduced competition for sunlight; hence, enough photosynthetic surfaces would be available to promote an increase in height (Adebayo and Akoun 2002). The number of leaves/plant in the mixed samples were significantly higher than in the sole because $C$. olitorius had higher height amongst the mixtures and tall plants have a faster rate of growth because they are less affected by the shading effect of neighbouring plants. These plants were thus able to trap the active radiation portion of the solar spectrum for photosynthesis more effectively. The height advantage further ensured the competitiveness of $C$. olitorius and subsequently its yield. The enhanced edible yield (leaves only) obtained in the mixed compared to sole cropping suggests that $C$. olitorius was able to compete with the neighbouring plants for available water, nutrients and light and able to utilize it effectively. In addition, the increased number of leaves is synonymous with an increased photosynthetic area and thus greater photosynthetic production, which translates into higher yield (Ibrahim 2002). Chowdury (1988) showed that the net returns from mixed crops of okra/ginger were higher than their 
monocrops. Ojeifo and Lucas (1987) reported that C. olitorius could be conveniently intercropped with another crop without adverse competition for incoming light. The impressive results obtained with mixed cropped $C$. olitorius are consistent with recent reports that mix cropping increases the size and stability of yields compared to sole cropping (Hauggard-Nielsen et al. 2006). Yield advantage of crops in mixtures often accrues from the capacity of the component species to increase the capture and use of biophysical resources relative to that achievable by growing the crops separately (Jahansooz et al. 2007). Yield increases with intercropping are associated with complimentary effects, better resource use efficiency and the buffering effects of the mixtures against diseases and weeds (Anil et al. 1998, Dapaah et al. 2003).

\section{Growth, development and yield of Corchorus olitorius as affected by plant extract}

There were improvements in the growth of C. olitorius treated with aqueous plant extracts. This may be due to a reduction in insect pests or the percentage infestation by the pests or both. The vegetables treated with plant extracts grew taller and had more leaves compared with the untreated control plants. The number of leaves/plant in the $A$. indica and $P$. guineense treated plants were higher than in the control. The results were comparable to those obtained for cypermethrin treated plants. This may be due to better protection from the various vegetable insect pests by the plant extracts. $A$. indica treated mixed cropped $C$. olitorius had higher values for edible yield and leaf area, which suggests that yield was influenced primarily by the level of competition with the neighbouring plants for available water, nutrients and light, which corroborate the findings of Montes-Molina (2008) in which mean yield was significantly higher in $A$. indica treated maize. The results from this research agreed with the findings of various authors on the efficacy of aqueous $A$. indica extract in reducing insect and pest damage in field crops (Mojumder et al. 2002, Javed et al. 2008).

\section{Growth and development of Corchorus olitorius as affected by mixed cropping and plant extracts}

The interaction effect of mixed cropping and plant extract treatment showed a strong positive relationship with the growth and yield parameters of $C$. olitorius. The strong performance with the treatment combination suggests that benefits associated with the use of the plant extracts and mixed cropping are complementary. While mixed cropping may enhance more efficient nutrient utilisation, plant extract treatment might have created an environment not suitable for insect growth and proliferation. The cropping system impacts soil properties, and by extension, their ability to support a good crop yield (Liebig et al. 2004, HauggaardNielsen et al. 2006, Jagadamma et al. 2008). A combination of other factors, namely tillage, crop sequence and cropping intensity, influence soil quality indicators and yield parameters (Liebig et al. 2004). Better resource use efficiency and the buffering effects of the plant extracts against diseases and weeds may have accounted for the increased yield in mixed cropped field crops (Anil et al. 1998). The use of these plant extracts thus has potential for use for improving the growth and yield performance of C. olitorius.

\section{Insect pest abundance under sole and mixed cropping}

Findings from this study support the general view that cropping systems have a reducing influence on insect pest infestations and associated crop damage. Mixed cropping of Cor $+\mathrm{Ama}+\mathrm{Cel}$ reduced pest infestation in comparison to $\mathrm{Cor}+\mathrm{Cel}$, Cor $+\mathrm{Ama}$ or Ama + Cel. On the other hand, sole cropping increased the pest density on the vegetables. The most abundant insect pests associated with the crops studied were Podagrica sp., Sylepta sp., Dsysdercus sp., and Zonocerus sp. The incidences of these insect pests were significantly reduced in mixedcropped $C$. olitorius. This is consistent with previous studies by Ogbalu et al. (2005) and Akinlosotu (1983). Ogenga-Latigo et al. (1992) demonstrated that intercropping beans with closely spaced maize reduced the incidence of $A$. fabae on beans. Similarly, Sinthanantham et al. (1990) observed a lower incidence of bean aphid on beans intercropped with maize than on sole cropped beans. Also, Edema et al. (1997) reported that aphid infestation was lower on intercropped than sole cropped cowpea.

\section{Insect pest abundance as affected by plant extracts}

The results of this study confirmed the insect control characteristics of the plants screened. The insect infestation rate was lower in sole and mixed cropped $C$. olitorius sprayed with aqueous plant extracts. A. indica and $P$. guineense extracts were effective in protecting the crops from the various insect pests, with values comparable to the synthetic insecticide $\left(\mathrm{Cymbush}^{\mathrm{R}}\right)$ used as a positive control. The presence of components capable of influencing 
the physiology and behaviour of a wide range of insects, mites and nematodes in $A$. indica and P. guineense extracts (Dhar et al. 1998, Schaaf et al. 2000, Ngono Ngane et al. 2003) may explain their efficacy in the control of insect pests infesting the vegetables studied. The major biologically active components reported in $A$. indica and $P$. guineense include: azadirachtin, triterpenoids, piperolein acids, salanine, nimbidin, guineensine, essential oils, sterols and amides, amongst others (Martins et al. 1998, Schaaf et al. 2000, Siddiqui et al. 2000, Muse et al. 2003, Ngono Ngane et al. 2003, Gopal et al. 2007). These components suppress some of the insects' desire for food and destroy eggs and immature insects.

\section{CONCLUSIONS}

1. Mixed-cropped $C$. olitorius had better growth and yield parameters than its sole-cropped counterpart.

2. Mixed cropping and plant extract sprays interacted positively to control insect pests and enhance yield.

3. The use of an aqueous extract of $A$. indica bark and $P$. guineense seed at concentrations $\geq 200 \mathrm{~g}$ $\mathrm{dm}^{-3}$ may give effective protection against insect pests infesting these vegetables and enhanced growth and yield comparable with the use of synthetic insecticides.

\section{REFERENCES}

Adebayo O., Akoun J., 2002. Effect of organic manure and spacing on the yield and yield components of A. cruentus. Proc. Annual Conference of the Horticultural Society of Nigeria (HORTSON). Edited by Umeh, V.C. and Fagbayide, J.A., Perfect Touch graphics Ibadan: 63-67pp.

Akendengue B., Lemamy G.J., Bourobou H.B., Laurens A., 2005. Bioactive natural compounds from medico-magic plants of the Bantu area. Studies in Natural Product Chemistry, Part 12, 32: 803-820.

Akinlosotu T.A., 1983. Destructive and beneficial insects associated with vegetables in South West Nigeria. Trop. Hort. 6: 217-228.

Anil L., PArK J., Phipps R.H., Miller F.A., 1998. Temperate intercropping of cereals for forage: a review of the potential for growth and utilization with particular reference to the UK. Grass Forage Sci. 53: 301-317.

Chowdury P.C., 1988. Intercropping short duration summer crops with ginger in the Darjelling Hills. Indian Farming 37(2): 4-5.
DapaAh H.K., Asafu-Agyei J.N., Ennin S.A., Yamoah C.Y., 2003. Yield stability of cassava, maize, soybean and cowpea intercrops. J. Agric. Sci. 140: 73-82.

Dhar R., Zhang K., Talwar G.P., Garg S., Kumar N., 1998. Inhibition of the growth and development of asexual and sexual stages of drug sensitive resistant strains of the human malaria parasite Plasmodium falciparum by $A$. indica (Azadirachta indica) fractions. J. Ethnopharmacol. 61: 31-39.

Edema R., Adipala E., Florini D.A., (1997). Influence of season and cropping system on occurrence of cowpea diseases in Uganda. Plant Disease 81: 465-468.

Eltun R., Korsæth A., Nordheim O., 2002. A comparison of environmental, soil fertility, yield, and economical effects in six cropping systems based on an 8-year experiment in Norway. Agric. Ecosys. Environ. 90: 155-168.

Georges K., Jayaprakasam B., Dalavoy S.S., Nair M.G., 2008. Pest-managing activities of plant extracts and anthraquinones from Cassia nigricans from Burkina Faso. Bioresource Technol. 99: 2037-2045.

Gopal M., Gupta A., Arunachalam V., Magu S.P., 2007. Impact of azadirachtin, an insecticidal allelochemical from $A$. indica on soil microflora, enzyme and respiratory activities. Bioresource Technol. 98(16): 3154-3158.

Han M.K., KIM S.I., AhN Y.J., 2006. Insecticidal and antifeedant activities of medicinal plant extracts against Attagenus unicolor japonicas (Coleoptera: Dermestidae) J. Stored Prod. Res. 42: 15-22.

HauggaArd-Nielsen H., Andersen M.K., JørnsgaArd B., JENSEN E.S., 2006. Density and relative frequency effects on competitive interactions and resource use in pea-barley intercrops. Field Crops Res. 95: 256-267.

IвRAнiм R., 2002. Correlation studies and percent contributions of different growth and yield characters to total fruit yield of tomato (Lycopersicon esculentum mill). Proc. Horticultural Society of Nigeria Conference: 28-32.

IJARotimi O.S., Eleyinmi A.F., Ifesan B.O.T., 2003. Evaluation of the nutritional status of adolescents in institutionalised secondary schools in Akure, Nigeria. Food Agric. Environ. 1(3,4): 64-68.

IKEORGU J.E.G., 1990. Glasshouse performance of three leafy vegetables grown in mixtures in Nigeria. Sci. Hort. 43(3): 181-188.

Ikeorgu J.E.G., Ezumah H.C., Wahua T.A.T., 1989. Productivity of Species in Cassava/Maize/Okra/ Egusi Melon Complex Mixtures in Nigeria. Field Crops Res. 21: 1-7.

Jagadamma S., Lal R., Hoeft R.G., Nafziger E.D., AdEE E.A., 2008. Nitrogen fertilization and cropping system impacts on soil properties and their relationship to crop yield in the central Corn Belt, USA. Soil Till. Res. 98: 120-129.

Jahansooz M.R., Yunusa I.A.M., Coventry D.R., Palmer A.R., Eamus D., 2007. Radiation- and water- 
use associated with growth and yields of wheat and chickpea in sole and mixed crops. Europ. J. Agron. 26: $275-282$.

Javed N., Gowen S.R., El-Hassan S.A., Inam-ul-HaQ M., Shahina F., Pembroke B., 2008. Efficacy of $A$. indica (Azadirachta indica) formulations on biology of root-knot nematodes (Meloidogyne javanica) on tomato. Crop Prot. 27(1): 36-43.

Jovanovic Z., Kostic M., Popovic Z., 2007. Grainprotective properties of herbal extracts against the bean weevil Acanthoscelides obtectus Say. Ind. crop Prod. 26: 100-104.

Liebig M.A., Tanaka D.L., Wienhold B.J., 2004. Tillage and cropping effects on soil quality indicators in the northern Great Plains. Soil Till. Res. 78: 131-141.

Martins A.P., Salgueiro L., Vila R., Tomi F., Cañigueral S., Casanova J., Proença da Cunha A., Adzet T., 1998. Essential oils from four Piper species. Phytochemistry. 49(7): 2019- 2023.

Mojumder V., Kamra A., Dureja P., 2002. Effect of $A$. indica extract on activity and mortality of second stage juveniles of Meloidogyne incognita. Nematol. Medit. 30: 83-84.

Mohammed Y., (2002): Farmers awareness building on Integrated Pest Management (IPM). Research report, ICIPE/EARO vegetable IPM project 16pp.

Montes-Molina J.A., Luna-Guido M.L., Espinoza-Paz N., Govaerts B., Gutierrez-Miceli F.A., Dendooven L., 2008. Are extracts of A. indica (Azadirachta indica A. Juss. (L.) and Gliricidia sepium (Jacquin) an alternative to control pests on maize (Zea mays L.). Crop Prot.. 27(3-5): 763-774.

Muse W.A., Lajide L., Adedire C.O., 2003. Effect of some Nigerian plants on survival, oviposition, and emergence of adult butterfly, Chrysomya chloropyga (Wied.) (Diptera: Callliphoridae). J. Asia-Pacfic Entomol. 6(1): 69-75.

Negi P.S., Roy S.K., 2001. Effect of drying conditions on quality of green leaves during long term storage. Food Res. Int. 34: 283-287.

Ngono Ngane, A., Biyiti L., Bouchet Ph., Nkengfack A., Amvam Zollo P.H., 2003 Antifungal activity of Piper guineense of Cameroon. Fitoterapia 74(5): 464-468.

Ngouajio M., McGiffen M.E. JR, Hutchinson C.M., 2003. Effect of cover crop and management system on weed populations in lettuce. Crop Prot. 22: 57-64.

Овон G., Akindahunsi A.A., 2004. Change in the ascorbic acid, total phenol and antioxidant activity of some sun-dried green leafy vegetables in Nigeria. Nutr. Health. 18: 29-36.

Ogbalu O.K., Amachree E.I., Amifor P.N., BenKalio G., 2005. The distribution of insect fauna of cultivated vegetables of the Niger Delta, Nigeria. App. Tropical Agriculture 9: 1-6.

Ogenga - Latigo M.W., Baliddawa C.W., Ampofo J.K.O., 1992. Influence of maize row spacing on infestation and damage of intercropped beans by bean aphids (Aphis fabae Scop.). 11. Reduction in bean yields. Field Crops Res. 30: 123-130.

OJeIfo I.M., LuCAS, E.O., 1987. The growth and development of Corchorus olitorius (L.) grown alone and intercropped with tomato (Lycopersicon esculentum mill). J. Agric. Sci. 109: 39-45.

Ojeifo I.M., Emuh F.N., Denton O.A., 2006. Crop production systems of market gardens in Nigeria. Eur. J. Soc. Sci. 2(2): 269-278.

Olasantan F.O., 1999. Nitrogen fertilization of okra (Abelmoschus esculentus) in an intercropping system with cassava (Manihot esculenta) and maize (Zea mays) in South-Western Nigeria. J. Agric. Sci. 133: 325-334.

Olasantan F.O., 2005. Cassava cultivation management for sustainable vegetable production in intercropping with okra. J. Sustain. Agric. 27(2): 53-68.

Oniango R.K., 2001. Enhancing People's nutritional status through revitalization of agriculture and related activities in Africa. Afr. J. Food Nutr. Sci. 1(1): 43-49.

Pavela R., 2004. Insecticidal activity of certain medicinal plants. Fitoterapia 75: 745-749.

Porter G., Harris F., Lyon F., Dung J., Adepetu A.A., 2003. Markets, ethnicity and environment in a vulnerable landscape: the case of small-scale vegetable production on the Jos Plateau, Nigeria, 1991-2001. Geogr. J. 169(4): 370-381.

PÜNTENER W., 1981. Manual for field trials in plant protection. Ciba-Geigy Limited, Basle, Switzerland: 205 pp.

SchaAf O., Jarvis A.P., van der Esch S.A., Giagnavoco G., Oldham N.J., 2000. Rapid and sensitive analysis of azadirachtin and related triterpenoids from $A$. indica (Azadirachta indica) by high performance liquid chromatography-atmospheric pressure chemical ionization mass spectrometry. J. Chromatogr. 886: 89-97.

Siddiqui B.S., Afshan F., Ghiasuddin S., Faizi S.N.H., NAQvi TARIQ R.M., 2000. Two insecticidal tetranortriterpenoids from Azadirachta indica. Phytochemistry 53: 371-376.

Sinthanantham S., Sohati P.H., Kaunatyan J., Haciwa H.C., 1990. Preliminary studies of bean aphid BCMV management in Zambia. Proc. $9^{\text {th }}$ SUA/CRSP and $2^{\text {nd }}$ SADCC/CIAT bean research workshop held at Sokoine University of Agriculture, Morogoro, Tanzania, 17-22 September, 1990. Bean Research 5: 162-168.

ThangataA P.H., Alavalapatib J.R.R., 2003. Agroforestry adoption in southern Malawi: the case of mixed intercropping of Gliricidia sepium and maize. Agr. Syst. 78: 57-71.

Received Februray 2, 2013; accepted March 25, 2013 